\title{
Análisis de los géneros periodísticos utilizados en los suplementos culturales españoles para comunicar la música clásica
}

\section{Analysis of Journalistic Genres used in Spanish Cultural Supplements to communicate classical music}

\begin{abstract}
Esther Martín Sánchez-Ballesteros. Universidad CEU San Pablo (esther.martinsanchezballesteros@colaborador.ceu.es)
\end{abstract}

\section{Resumen:}

En esta nota de investigación se analizan los géneros periodísticos más utilizados por los suplementos culturales Babelia, ABC Cultural y El Cultural para comunicar los contenidos de música clásica entre 1991 y 2010. El objetivo es investigar la evolución de los estilos y géneros más comunes de esta sección al pasar de la última década del siglo XX a la primera del XXI. A través de un análisis cuantitativo se han obtenido unos resultados y líneas de tendencia en los que se observa una clara inclinación hacia el uso de los géneros interpretativos con la reseña como principal protagonista. Sin embargo, el uso generalizado del artículo de opinión en los últimos años indica un posible cambio en las prácticas periodísticas.

Palabras clave:

Géneros periodísticos, música clásica, Babelia, El Cultural, ABC Cultural, suplementos culturales.

\begin{abstract}
:
This article discusses the journalistic genres most commonly used by the cultural supplements Babelia, ABC Cultural and El Cultural to communicate the contents of classical music from 1991 to 2010. The aim is to investigate the evolution of most common styles and genres of this section from the last decade of the 20th Century to the first of the 21th. The results and the trend lines show a clear leaning towards the use of interpretive genres, with the review article as the main protagonist, have been obtained through quantitative analysis. However, the widespread use of the opinion article in recent years indicates a possible change in the journalistic practices.
\end{abstract}

Keywords:

Journalistic genres, classical music, Babelia, El Cultural, ABC Cultural, cultural supplements 


\section{Introducción}

Esta investigación pretende constatar la situación en la que se encuentra la música clásica en el periodismo impreso tras su paso del siglo XX al XXI. El análisis aporta datos esclarecedores sobre las diferentes prácticas que los diarios de referencia, a través de sus suplementos de cultura, han hecho de los estilos y géneros periodísticos. El alcance de la investigación abarca otros aspectos que se han dejado fuera del presente artículo.

\section{Hipótesis y objetivos de la investigación}

La hipótesis de partida plantea el deterioro de la información especializada en música clásica conforme se aleja el siglo XX y avanza el XXI.

Para demostrarlo se propone el objetivo de analizar diversos aspectos de los géneros periodísticos en la sección de música clásica de estas publicaciones.

\section{Marco teórico de la investigación: Periodismo especializado}

Uno de los principios de este estudio es incluir la música clásica en el periodismo especializado y huir de la mera crítica. Para llegar a una aproximación de este concepto se parte de la Información Periodística Especializada de Fernández del Moral y Esteve (1996: 100): "La IPE es aquella estructura informativa que penetra y analiza la realidad de una determinada área de la actualidad a través de las distintas especialidades del saber; profundiza en sus motivaciones; la coloca en un contexto amplio, que ofrezca una visión global al destinatario, y elabora un mensaje periodístico que acomode el código al nivel propio de la audiencia, atendiendo a sus intereses y necesidades".

En lo que se refiere al periodismo especializado en música clásica, en España tardó tiempo en llegar. Aunque el compositor alemán Telemann creó la gaceta quincenal Der Getreue Musik-Meister (El amistoso maestro de música) en 1728 no será hasta 1819 cuando se publica la primera crítica musical en El Diario de Barcelona ${ }^{1}$.

El Diario de Barcelona se publicó por primera vez en 1792 y abogaba por una ideología monárquica y liberal-conservadora que a la larga le hizo perder influencia. 


\section{Estado de la cuestión}

\subsection{Periodistas españoles de referencia en el siglo XX}

Durante el siglo XX, el periodismo musical experimenta un enorme desarrollo en España, representado por cuatro grandes profesionales²: Adolfo Salazar, Padre Federico Sopeña, Antonio Fernández-Cid y Enrique Franco.

\subsection{Revistas especializadas en música clásica y otros medios}

España tiene una amplia tradición en el ámbito de las revistas especializadas: Ritmo, fundada en 1929; Squerzo, en 1985; Ópera Actual, en 1991 y Melómano en 1996. En otros medios la representación de la música clásica es escasa: no existe ningún canal de televisión español dedicado a la música clásica; en la radio, la consolidada Radio Clásica, y en Internet, vigentes en 2010, Mundoclasico.com, Codalario.com, Docenotas.com y Clasica2.com.

\subsection{Hábitos de consumo de la música clásica}

El Ministerio de Educación, Cultura y Deporte elabora periódicamente la "Encuesta de Hábitos y Prácticas Culturales en España".

Tabla 1: Hábitos de consumo de la música clásica entre 1990-2011

\begin{tabular}{|c|c|c|c|c|c|}
\hline \multirow{2}{*}{ 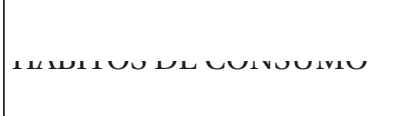 } & \multicolumn{5}{|c|}{ Personas que asisten a dicha actividad } \\
\hline & 1990 & $1997-1998$ & 2002-2003 & 2006-2007 & 2010-2011 \\
\hline Ópera & $1,4 \%$ & $1,8 \%$ & $3 \%$ & $2,7 \%$ & $2,6 \%$ \\
\hline Zarzuela & $2,7 \%$ & $2,2 \%$ & $2,6 \%$ & $1,9 \%$ & $1,6 \%$ \\
\hline \multicolumn{6}{|l|}{ Conciertos } \\
\hline \multirow[t]{2}{*}{ Música Clásica } & $6,8 \%$ & $6,8 \%$ & $8,4 \%$ & $8,4 \%$ & $7,7 \%$ \\
\hline & \multicolumn{5}{|c|}{ Otras variables de consumo } \\
\hline Lectura Revistas Culturales & No ha & & $13,2 \%$ & $15,2 \%$ & $17,7 \%$ \\
\hline
\end{tabular}

Aunque se pueden echar en falta algunos nombres, como el de Xavier Monsaltvage, los expertos consultados coincidieron en su preponderancia. 


\section{Delimitación del universo de estudio}

Con el fin de constatar la hipótesis de partida se ha realizado un análisis en base a tres elementos: los suplementos de la prensa de referencia, el territorio español y el paso del siglo XX al XXI.

\subsection{Suplementos}

Los suplementos escogidos como universo de la muestra pertenecen a la prensa de referencia, es decir, "aquellos medios informativos cuyas noticias y comentarios se convierten en fuente de referencia internacional o nacional para otros medios informativos" (Núñez Ladevéze, 1995: 31): Babelia de El País, ABC Cultural de ABCy El Cultural de El Mundo.

\subsection{Delimitación geográfica y cronológica}

La comunicación musical es un filón estratégico para observar la transformación cultural de la sociedad, de ahí que la selección se publique en todo el territorio español. Además, con la intención de ofrecer una visión panorámica del final de siglo XX y comienzo del XXI, los suplementos escogidos nacieron en la década de los 90 y se han publicado hasta el 31 de diciembre de 2010. Siguiendo a los investigadores Wimmer y Dominick (1996: 73) y su "sistema aleatorio sistemático", los años analizados son 1991, 1995, 1998 (sólo para El Cultural), 2000, 2005 y 2010.

\subsection{Muestra total}

La muestra total está compuesta de 574 ejemplares. Desde un punto de vista cuantitativo resulta satisfactoria porque supera las escasas investigaciones realizadas hasta la fecha y representa científicamente el universo total de la materia de estudio.

\section{Metodología}

Para asegurar la fiabilidad y veracidad de los datos se ha planteado una triangulación de métodos: entrevistas en profundidad y análisis de contenido. Es importante resaltar que las entrevistas se realizaron a once informantes clave, "personas respetadas y conocedoras en su profesión” (Taylor y Bogdan, 1994: 61). Juan Ángel Vela del Campo, Joachim Pflieger, Álvaro Guibert, Stefano Russomanno, Benjamín G. Rosado, Gonzalo Alonso, Arturo Reverter, Fernando Sans, Xoan María Carreira, José Luís Ga del Busto y Alberto González.

En el análisis de contenido se han trabajado dos tipos de variables obtenidas a partir de las entrevistas: cualitativas, para profundizar en el contenido, y cuantitativas, para obtener la evolución del género periodístico. Para delimitarlas correctamente se siguieron los principios que evitan el solapamiento entre las categorías: "Exclusión mutua, homogeneidad, pertinencia, objetividad y fidelidad" (Bardin, 1996: 135). 


\section{Delimitación de los géneros periodísticos}

\subsection{Géneros informativos}

Reportaje informativo. Según Martínez Albertos es el "relato periodístico de una cierta extensión y estilo literario muy personal en el que se intenta explicar cómo han sucedido unos hechos actuales o recientes” (1991: 302).

Información. Coincide con el concepto popular de noticia y "la forma literaria más escueta para presentar una noticia" (Martínez Albertos, 1991: 288).

\subsection{Géneros interpretativos}

Crónica. Presupone una cierta regularidad por parte del periodista y "se sitúa más cerca del ámbito de la crítica o de la reseña que del de la noticia" (Rodríguez Pastoriza, 2006: 123).

Reportaje interpretativo. Ha de fundamentarse en hechos recientes e incluso desconocidos, "aunque no sean rigurosamente actuales pero sí de interés para la sociedad" (Martínez Albertos, 1991: 331).

Entrevista. "Resulta un género muy adecuado para el periodismo cultural escrito por la posibilidad del periodista de introducir acotaciones explicativas” (Rodríguez Pastoriza, 2006: 125).

Reseña. En el proceso de esta investigación se apreció que eran los datos y la interpretación que hacía el redactor lo que conformaban este género en cuanto a música clásica.

\subsection{Géneros de opinión}

Editorial. Se encomienda a una voz relevante del periódico y vierte opiniones personales sobre un tema de actualidad.

Artículo. Se basa en la exposición de ideas surgidas a propósito de hechos recientes.

Columna. La realiza un colaborador habitual del periódico que comenta un tema y "cuya libertad se nos revela como un aspecto esencial de la libertad de comunicar los pensamientos” (Linares, 2011: 130).

Crítica. Es el artículo que analiza una pieza cultural o artística teniendo presente que "criticar no es censurar" (Santamaría, 1997: 144). 


\section{Respuestas de los Informantes Clave respecto al uso de géneros:}

- Gonzalo Alonso. Colaborador de El Cultural y La Razón: "En mi caso escribo todo tipo de géneros, pero fundamentalmente hago crítica. En ocasiones me ofrecen escribir sobre algún tema en concreto, pero fundamentalmente soy yo el que elijo".

- Xoan María Carreira. Fundador y redactor jefe de Mundoclasico.com: "Respecto a los géneros, lo normal es que cada espectáculo lo exija por sí mismo, pero si el periodista tiene una opinión diferente se propicia un acuerdo y se llega a él con suma facilidad. Mundoclásico.com funciona con la lógica de cualquier diario: cada género lo va dando la propia actualidad".

- José Luís García del Busto. Colaborador de El País y El Cultural: "A los periódicos les da igual el género que se utilice e incluso la cantidad de textos que se entreguen: si mandas algo lo publican, y si no, no importa mucho [...]Personalmente, he hecho pocas entrevistas o reportajes porque consideraba que no era mi campo".

- $\quad$ Benjamín García Rosado. Redactor de El Cultural: "En el suplemento hay un equilibrio entre la distribución de los géneros: primero entre la sección misma, cuidamos que haya un reportaje, un artículo, entrevista, debate... Después entre las demás secciones del suplemento".

- $\quad$ Alberto González Lapuente. Colaborador de $A B C$ y Coordinador de actividades de la Fundación Guerrero: "Fundamentalmente siempre he hecho crítica y en contadas ocasiones he redactado algún artículo más generalista. En cualquier caso, siempre me lo encargan con el género especificado desde el propio periódico".

- $\quad$ Álvaro Guibert. Director de contenidos de la Fundación Albéniz y colaborador de El Mundo: "Elegir los géneros es soberanía del dueño del periódico y siempre la he respetado".

- $\quad$ Arturo Reverter. Colaborador con El Cultural, La Razón y la revista Scherzo: "Generalmente hago lo que me dicen respecto a los géneros periodísticos, aunque siempre está la posibilidad de sugerir. En alguna ocasión he rechazado realizar entrevistas porque no es una cosa que me entusiasme".

- $\quad$ Stefanno Russomanno. Redactor de ABC Cultural: "Yo decido los géneros y con el paso del tiempo he ido cambiando mis propias normas. El criterio va evolucionando, porque tú mismo cambias y por razones externas que tienen que ver con el número que disponga de páginas. Ahora, al tener poco espacio para la sección, escojo temas que no tratan ni el periódico ni otros suplementos".

- $\quad$ Fernando Sans. Director de Ópera Actual: "Nosotros utilizamos todos los géneros [...] Nuestro producto se diferencia en eso, en abarcar todos los campos, es muy importante. Intentamos que siempre haya variedad de géneros y de información". 
- Juan Ángel Vela del Campo. Comentarista de la Cadena SER y colaborador de El País: "El mayor problema respecto a los géneros es que la crítica va a desaparecer de los periódicos y sólo se utilizará en circunstancias muy excepcionales. Ya no interesa la crítica del especialista en general, ni del de música en particular. En cuanto al suplemento de cultura, el lector no tiene las suficientes referencias y su comprensión naufraga. [...] evito la entrevista, si la puede hacer alguien del periódico, mejor. En cuanto a la reseña y la crónica tienen un toque mixto: hoy día se hace un tipo de reseña que es más bien una crónica, aunque el género de la crónica domina sobre la reseña".

\section{Resultados del análisis de contenido}

\subsection{Espacio dedicado a la música clásica}

En esta investigación se refleja que la presencia y variedad de diversos géneros periodísticos en los suplementos depende, en gran medida, de las páginas que se dediquen a música clásica. Al observar la distribución de las páginas que ha ocupado la música clásica entre 1991 y 2010 (tabla 2) se hace patente una paulatina disminución del espacio dedicado a esta sección, lo que propicia géneros de extensión más corta que, a su vez, favorecen la búsqueda de diferentes formatos. Es significativo que en un principio la música clásica ocupara el 23\% de las páginas de ABC Cultural y el $8 \%$ de Babelia para pasar a final del periodo de estudio a un $4 \%$ y un $2 \%$, respectivamente; esto indica que la disminución es del $83 \%$ en el suplemento de $A B C$ y del $75 \%$ de El País. En el caso de El Cultural los resultados son más esperanzadores, aunque evidencian nuevamente cómo la música clásica va desapareciendo de sus páginas: de un 12\% en 1998 pasa a ocupar el 6\% en 2010 , por lo que la disminución es del $50 \%$ en todo el periodo. Ante este hecho veremos más adelante que los periodistas se adaptan al espacio y acoplan los contenidos al género más adecuado.

Tabla 2: Porcentaje de páginas dedicadas a la música clásica

\begin{tabular}{|c|c|c|c|c|c|c|}
\hline \multirow{2}{*}{ SUPLEMENTO } & \multicolumn{6}{|c|}{ PÁGINAS DEDICADAS A M. CLÁSICA \% } \\
\hline & 1991 & 1995 & 2000 & 2005 & 2010 & TOTAL \\
\hline Babelia & $6,8 \%$ & $4,5 \%$ & $5,8 \%$ & $3,4 \%$ & $1,7 \%$ & $3,9 \%$ \\
\hline ABC Cultural & $24,3 \%$ & $13,2 \%$ & $13,4 \%$ & $8,1 \%$ & $3,5 \%$ & $10,6 \%$ \\
\hline \multirow{2}{*}{ El Cultural } & \multirow{2}{*}{$\mathrm{X}$} & 1998 & \multirow{2}{*}{$10,1 \%$} & \multirow{2}{*}{$10 \%$} & \multirow{2}{*}{$6,9 \%$} & \multirow{2}{*}{$9,3 \%$} \\
\hline & & $11,6 \%$ & & & & \\
\hline PROMEDIOS & $16,3 \%$ & $10,9 \%$ & $10,8 \%$ & $8,1 \%$ & $4,6 \%$ & $8,9 \%$ \\
\hline
\end{tabular}

Fuente: Elaboración Propia 


\subsection{Géneros informativos}

Reportaje Informativo: En total, el 22\% de la muestra incluía reportaje informativo. Es un porcentaje relevante si se tiene en cuenta que para Rodríguez Pastoriza este es uno de los géneros más creativos y que presenta mayores posibilidades de expresión a los profesionales del periodismo cultural. Además, es interesante observar el paso de una década a otra, pues si bien el reportaje informativo se consideró un género más importante durante los 90 cuando se incluyó en el $63 \%$ de los suplementos, con el comienzo del siglo XXI su presencia disminuye hasta el 20\%. Aunque su aparición es heterogénea a lo largo del periodo, todo parece indicar una recuperación del género en dos de los suplementos estudiados, Babelia y El Cultural. En cuanto al suplemento con mejores resultados, El Cultural es el que más reportajes informativos publica con una cifra superior a los otros dos (51\%).

Información. Tanto ABC Cultural como El Cultural consideran este género fundamental en el origen de la publicación, por lo que aparece en el 100\% de ellos; después mantienen una presencia evidente, aunque de diferente manera. Durante las dos décadas que analiza este artículo, El Cultural es el suplemento que incluye la información más veces (85\%). Al comparar su uso entre una década y otra, vemos que los datos formarían figurativamente una línea cóncava que tendría como punto de inflexión el año 2000, el más notable para este género, pues está presente en el 59\% de la muestra. Además, el 44,8\% de los suplementos entre 1991 y 2010 lo contenía, pero su presencia disminuye notablemente entre los años 2000 y 2010, cuando aparece en un 73\% en el caso de ABC Cultural y un 27\% en el de El Cultural. Babelia es el único que la aumenta en un $1 \%$, una cifra pequeña y poco significativa.

Tabla 3: Evolución de los géneros informativos entre 1991 y 2010

\section{GÉNEROS INFORMATIVOS}

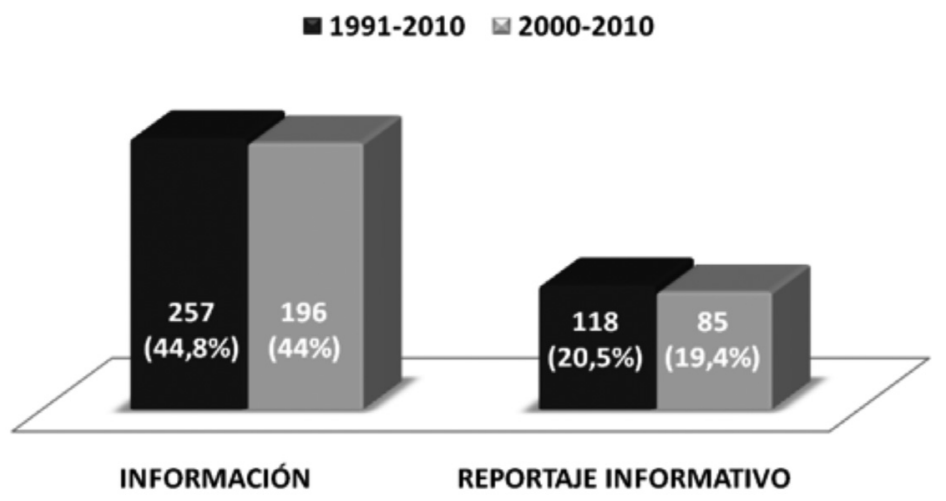

Fuente: Elaboración propia 


\subsection{Géneros interpretativos}

Reseña: Su utilización está muy extendida entre los suplementos culturales, que la incluyeron en el 67,7\% de las ocasiones, y los datos presentan unas grandes similitudes entre unas y otras cabeceras. Así, ABC Cultural vuelve a ser el que más veces recurra a dicho género, con un total del 86,9\%. Tanto ABC Cultural como El Cultural lo incluyeron en el 100\% de sus primeras publicaciones. Las cifras obtenidas en el año 2000 lo elevan al número uno en presencia total, pues se halla en el 90\% de los suplementos analizados. Lamentablemente, los resultados negativos de 2010 rompen las líneas de comportamiento que se habían mantenido tanto a finales del siglo XX como a principios del XXI.

Crónica: Las escasas veces que hace aparición (1\%) son posteriores al año de nacimiento de cada suplemento. Los dos que la utilizan tienen un comportamiento muy similar, ABC Cultural en el siglo XX y Babelia en el XXI. Es "un género ideal para los programas informativos que ofrece la televisión dada su flexibilidad temporal” (Rodríguez Pastoriza, 2006: 123), cualidad poco adecuada en el suplemento por su periodicidad semanal.

Reportaje interpretativo: Se mantiene una línea de acción paralela y aparece en el origen de todas las publicaciones, con una presencia del 19,3\% en el conjunto de la muestra. Sin embargo, su evolución y el paso de un siglo a otro confirman el descenso de los datos hasta el final. Si nos fijamos en la cantidad de suplementos que contienen reportajes en un año determinado, 2005 es el que tiene el mayor número, el 28,7\% de la muestra. Entre las dos décadas que analiza esta investigación, El Cultural es el que mayor porcentaje de usabilidad tiene respecto a este género, con un 26,2\% y ABC Cultural le sigue muy de cerca (25\%).

Entrevista: Gracias al análisis se deduce un gran cambio entre las dos décadas respecto al uso de la entrevista. Durante sus primeros años de vida, los tres suplementos la tienen muy en cuenta, incluyéndola con frecuencia hasta el año 2000, cuando se encuentra en 58,7\%. El avance de la primera década del siglo XXI supone un giro en las redacciones, que dejan de incluirla. ABC Cultural lo hace de forma radical mientras que Babelia y El Mundo van abandonándolo progresivamente. De esta manera, en 2010 la encontramos en el 25\% de los números analizados. En la muestra global, El Cultural es el que presenta un mayor porcentaje de entrevistas en la totalidad del estudio, con un 66,9\% frente al 54,4\% del ABC Cultural y el 14,8\% de Babelia. Es una cifra elevada, del 47,7\%, lo que lo convierte en un género muy popular en esta sección. 
Tabla 4: Evolución de los géneros interpretativos entre 1991 y 2010

\section{GÉNEROS INTERPRETATIVOS}

—1991-2010 $=2000-2010$

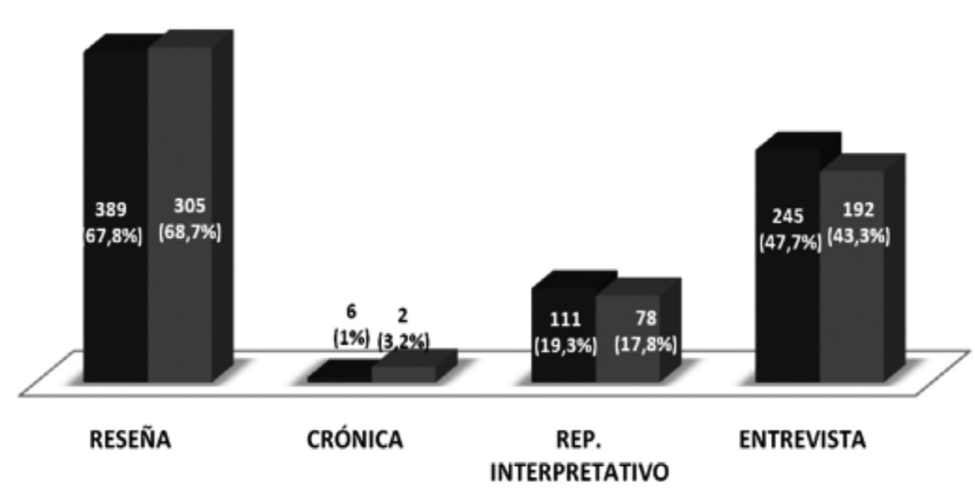

Fuente: Elaboración propia

\subsection{Géneros de opinión}

Artículo: De todos los suplementos publicados entre 1991 y 2010, el 45,8\% incluía el artículo; una cifra elevada. ABC Cultural es el suplemento que más veces opta por él, sus números no bajan nunca del $40 \%$. Obsérvese cómo la actitud respecto a este género en cada década es muy similar: en el inicio de los 90, el artículo ocupaba un lugar destacado y luego disminuye; lo mismo ocurre con la llegada del nuevo siglo, cuando las redacciones se preocupan de que esté presente entre sus páginas para terminar en 2010 apartándolo de nuevo. 2005 es el año que más cantidad de números incluyen el artículo, en concreto el 56\%. Dado que el artículo periodístico cuenta con una gran tradición en España, es relevante enfatizar los resultados, pues confirman el gusto que los redactores y colaboradores tienen por él.

Columna: Se encuentra en el 45,6\% de los ejemplares, por lo que los datos lo acercan a los valores del artículo y la información, con un 46\%. Entre 1991 y 2010 ABC Cultural es el que más veces incluye la columna; el 80\% de las ocasiones la redacción la ha programado entre sus páginas. Le sigue El Cultural, con el 74\%. De nuevo, Babelia se aleja de estos porcentajes con unas cifras mínimas que suponen el $1 \%$ del total. En cuanto al paso de un siglo a otro, la década de los 90 está marcada por una alta presencia de la columna en las páginas de música clásica de los suplementos, manteniendo un porcentaje superior al 95\% tanto en El Cultural como en ABC Cultural; la década siguiente supone un descenso brusco de su utilización, de un $98 \%$ y un $77 \%$ respectivamente. 
Crítica: El porcentaje de suplementos que ha incluido la crítica entre los años 1991 y 2010 es de un 4,3\%, la segunda peor cifra después del editorial, que era del $0 \%$ para toda la muestra. Al unir los resultados de las dos décadas se aprecia que es la segunda la que destaca por la inclusión del género en un total del $4 \%$, frente al $1 \%$ durante los 90 . Es una diferencia pequeña en puntos porcentuales, aunque significativa, porque demuestra que en su origen este género se consideraba prescindible, tendencia que no cambia significativamente hasta el 2005 , cuando se utiliza un $12 \%$ sobre la muestra. Será El Cultural el que en 2005 lo incluya en un 29\% de sus números, seguido de ABC Cultural, con un 10\% en 1995 y un $8 \%$ en 2005. El resto de cifras resulta muy pequeño con respeto al tamaño de la muestra, por lo que su representatividad es insuficiente para tenerse en cuenta.

Tabla 5: Evolución de los géneros de opinión entre 1991 y 2010

\section{GÉNEROS OPINIÓN}

—1991-2010 $=2000-2010$

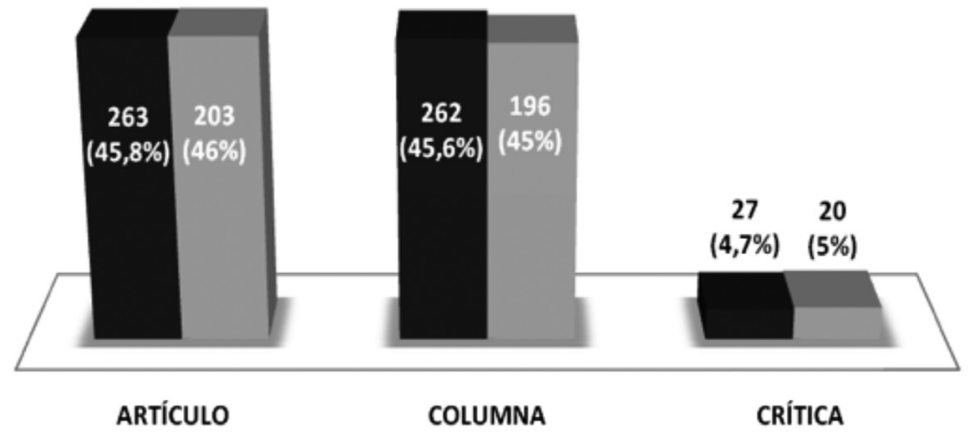

Fuente: Elaboración propia

\section{Conclusiones}

Los datos del trabajo de campo demuestran que la música clásica, en su proceso de adaptación como una sección diferenciada dentro de la oferta de los suplementos, se ha servido de todos los géneros periodísticos, a excepción de:

a. Los que necesitan una relación directa con la inmediatez (crítica, crónica).

b. Los que ocupan un lugar determinado dentro de la publicación (el editorial), que abren con un tema de actualidad relacionado con la cultura en general. 
Además, la música clásica en ellos se presenta mayoritariamente a través de los géneros interpretativos, seguidos de cerca por los informativos. A pesar de que en este tipo de publicación están representados todos los géneros, la aparición de nuevos soportes y la modificación del modelo de comunicación tradicional han incidido en la prensa escrita de manera directa, propiciando una búsqueda de la fórmula idónea para la supervivencia de este tipo de publicaciones. Si bien la transformación se produce de manera lenta a lo largo de las dos décadas, hemos observado que el año 2000 se presenta como punto de inflexión respecto a las estrategias seguidas en cada redacción. En lo referente a la cantidad de páginas que ocupa, el interpretativo se erige como el estilo más utilizado, con un 36,7\% de presencia sobre el total de la muestra (lo que se complementa con líneas de tendencia ascendente). La información, con un 36,6\%, le sigue de cerca, aunque su futuro es más incierto (sus líneas de tendencia son negativas).

Pese a que las variaciones no son grandes, lo realmente llamativo es su tendencia al crecimiento. Los resultados son propios de la coyuntura que atraviesa el periodismo en la actualidad y coinciden plenamente con las investigaciones de los expertos, cuando afirman que vivimos un cambio de paradigma: la clasificación convencional de los géneros está obsoleta y ahora "se cuestionan o se niegan principios como la objetividad, la separación entre información y opinión y la clásica teoría de los géneros basada en tales principios” (Herrero, 2004: 181).

En concreto, la reseña es el género periodístico más utilizado para comunicar la música clásica de los suplementos culturales. En la muestra total ha estado presente en el 67,8\% de los suplementos en los que había sección de música clásica, cifra que aumenta un punto porcentual durante la primera década del siglo XXI. La variedad de tamaños que permite este género, además del interés que provocan los nuevos materiales publicados, ya sean discos o libros, lo han convertido en el más popular, a lo que se suma que no sea necesaria la plena actualidad del hecho noticioso. Por otro lado, la reseña permite que el lector conozca tanto la opinión del periodista como la información sobre el producto, y no le exige niveles profundos de conocimiento en la materia para entender su contenido. Por suplementos, tanto en Babelia (52\%) como en ABC Cultural (87\%) la reseña es el género más usado, mientras que en El Cultural se sitúa en la tercera posición $(70,8 \%)$.

Le sigue la entrevista, el segundo en presencia con un total de 47,7\% de los números analizados que lo incluyó, y, en particular, fue El Cultural, con un amplio margen sobre los otros dos, el que presenta el mayor índice $(71,4 \%)$ de uso en este género.

En la primera década del siglo XXI el artículo de opinión es el género que experimenta un mayor crecimiento. Un total del $45,8 \%$ lo incluyó durante todo el estudio. El dato refleja que es el género que más aumenta su presencia en los suplementos culturales de la prensa de referencia española. Si durante la última década del siglo XX la encontramos en el $45 \%$ de los números estudiados, durante el siglo XXI aumenta un punto porcentual y asciende al $46 \%$. Su éxito pone de manifiesto la vinculación al periodismo de tradición clásica y el gusto de los suplementos culturales por la opinión de una figura reco- 
nocible, que en algunos casos puede ser un músico de renombre o un crítico conocido. Tales son los casos de la relación de colaboración que mantuvo el compositor Tomás Marco con ABC Cultural o la del crítico Juan Ángel Vela del Campo con El País.

En contraposición, la crítica desaparece de la sección de música clásica. Hasta la década de 1990 la información referente a la música clásica que se publicaba en los diarios y cuadernillos culturales estaba redactada por el llamado "crítico". Ellos tenían (Armañanzas, 1993: 160) "la función de la valoración argumentada de las obras de creación intelectual en la que el crítico nos ofrece una orientación y una evaluación crítica sobre esa elaboración [...]”.

Estos resultados evidencian la evolución de los géneros en busca del modelo más adecuado de periodismo especializado en música clásica y el formato de los suplementos como un marco propicio para la potenciación de las diferentes áreas del periodismo especializado. Si continuamos por este orden de popularidad, los cinco primeros son los que aparecen reflejados en la siguiente tabla:

Tabla 6: Géneros periodísticos más frecuentes en la música clásica

\begin{tabular}{|l|l|}
\hline GÉNERO PERIODÍSTICO & PRESENCIA EN \% \\
\hline Reseña & $67,8 \%$ \\
\hline Entrevista & $47,7 \%$ \\
\hline Artículo de opinión & $45,8 \%$ \\
\hline Columna & $45,6 \%$ \\
\hline Información & $44,8 \%$ \\
\hline
\end{tabular}

Fuente: Elaboración propia

Si se atiende al número de páginas, durante el año 2010 las dedicadas a la música clásica sufren una mayor disminución. En términos generales, el número de páginas de cada suplemento se ha reducido paulatinamente y unas temáticas han sustituido a otras; pero en lo que a música clásica se refiere, la disminución ha sido exponencial, llegando incluso a desaparecer en 2010. Los datos demuestran que $A B C$ Cultural presenta la mayor diferencia entre su origen y el final de la investigación, con una disminución del 20,8\% en su espacio dedicado a la música clásica. El Cultural (con el 9,3\%) y Babelia $(3,9 \%)$ se sitúan por detrás, a gran distancia la una de la otra.

Esto afecta directamente a los géneros: en un primer momento el proceso de asimilación de los géneros periodísticos fue muy rápido y todos estaban representados en menor o mayor medida en las secciones de música clásica pero, al disminuir el espacio, las redacciones tuvieron que decidir qué textos publicar y con qué forma. Si a esto se le añade la llegada de un nuevo medio de comunicación, Internet, la profesión adopta una perspectiva diferente que cuenta con estas dos va- 
riables (espacio y medio) para buscar un nuevo tratamiento de los géneros periodísticos con los que diferenciarse como soporte.

\section{Referencias bibliográficas}

Adorno, T. W. (2009): Disonancias. Introducción a la Sociología de la Música. Madrid: Akal.

Armañanzas, E. (2009): La crítica de las artes en los suplementos culturales.

AA.VV. (2009): Espéculo. Revista de estudios culturales. Madrid: Universidad Complutense, pp. 1-18.

Bardin, L. (1996): El análisis de contenido. Madrid: Akal.

Borrell Vidal, J. (1945): Sesenta años de música (1876-1936). Madrid: Dossat.

Carredano, C. (2004): Adolfo Salazar en España. Primeras incursiones en la crítica musical: la Revista Musical Hispano-Americana (1914-1918). Revista Anales del Instituto de Investigaciones Científicas, 106 (84), 119-144.

Casares Rodicio, E. (1995): La crítica musical en el XIX español. Panorama general. La música española en el Siglo XIX (pp. 465-497). Gijón: Universidad de Oviedo.

Fernández del Moral, J. y Esteve Ramírez, F. (1996): Fundamentos de la información periodística especializada. Madrid: Síntesis.

Flick, U. (2004): Introducción a la investigación cualitativa. A Coruña: Morata.

Halffter, C. (1982): El español y la música clásica. Anuario El País, pp. 181.

Hernández Sampieri, R., Fernández Collado, C. \& Baptista Lucio, P. (2010): Metodología de la Investigación. México D.F: Mac Graw Hill.

Herrero, C. (2004): Géneros para la divulgación periodística, en AA.VV. (2004): Periodismo especializado. Madrid: Ariel Comunicación, pp. 171-194.

Linares Rodríguez, Virginia (2011): La columna periodística, en AA.VV. Géneros y discurso periodístico. Madrid: Fragua, pp. 103-132.

Maciá, J. (1993): La comunicación regional y local. Madrid: Ciencia 3.

Moya Martínez, M. delV. (1997): Aproximación a la crítica musical madrileña del último tercio del siglo XIX. Ensayos 30(10), 163-171. 
Martínez Albertos, J. L. (2001): Curso general de redacción periodística: lenguaje estilos y géneros periodísticos en prensa, radio, TV y cine. Madrid: Paraninfo-Thomson Learning.

Núñez Ladevéze, L. (1995): Introducción al periodismo escrito. Barcelona: Ariel Comunicación.

Rodríguez Pastoriza, F. (2006): Periodismo Cultural. Madrid: Síntesis.

Santamaría Suárez, Luisa (1997): Géneros para la persuasión en periodismo. Madrid: Fragua.

Taylor, S. J. \& Bogdan, R. (1994): Introducción a los métodos cualitativos. Barcelona: Paidós.

Valera Cases, A. (1985): Cruz y drama de la música. Madrid: Alpuerto.

Wimmer, R. D. \& Dominick, J. R. (1996): La investigación científica de los medios de comunicación. Una introducción a sus métodos. Barcelona: Bosch Casa Editorial. 